This is an Accepted Manuscript of an article published by Emerald Publishing Limited in Working with Older People on 22 October 2019 available at https://doi.org/10.1108/WWOP-09-2019-0023

\title{
Citation:
}

Anantapong, K. and Tinker, A. (2019), "Attitudes towards frailty assessment in clinical practice among psychiatrists in the UK", Working with Older People, Vol. 23 No. 4, pp. 185194. https://doi.org/10.1108/WWOP-09-2019-0023

\section{Title: Attitudes towards frailty assessment in clinical practice among psychiatrists in the UK}

Kanthee Anantapong a,b ${ }^{\text {a }}$ MD, FRCPsychT, MSc

Anthea Tinker ${ }^{\text {a }}$, CBE, Ph.D, FKC, FAcSS, FRSA

a Department of Global Health and Social Medicine, School of Global Affairs, Faculty of Social Science and Public Policy, King's College London, London, UK, WC2B 4BG

b Department of Psychiatry, Faculty of Medicine, Prince of Songkla University, Hat Yai, Songkhla, Thailand, 90110

Corresponding author: Kanthee Anantapong

Postal address: Department of Psychiatry, Faculty of Medicine, Prince of Songkla University, Hat Yai, Songkhla, Thailand, 90110

Email address: kanthee.anan@gmail.com

Twitter: @KantheeMD 


\begin{abstract}
Purpose: Although there is substantial evidence about the association between frailty and mental illnesses in older people, there is currently little evidence about how this is integrated into psychiatric clinical practice. The aim of this research is to explore the attitudes of a sample of psychiatrists in the UK about the concept and assessment of frailty in their clinical practice.
\end{abstract}

Design/methodology/approach: This research used a qualitative approach with semistructured interviews. Interview schedules and transcripts were analysed using thematic analysis. NVivo software and an audit trail were used for the data analysis.

Findings: There were 10 respondents (all psychiatrists) in this study. From the interviews, it appeared that some respondents might not be fully familiar with existing concepts of frailty. However, from their perspectives, frailty appeared to be multi-dimensional, reversible, and for some could be enhanced by older people's ability. Negative stereotypes of being frail could undermine the accessibility to appropriate assessment and care. Existing multidisciplinary assessments, with some adjustments to the particular needs of psychiatric patients, can be used to establish a frailty index.

Originality/value: The concept of frailty, especially those concerning its multidimensional and homeostatic nature, should be further examined to make it more applicable to psychiatric practice. Without much more effort, frailty could be assessed within current psychiatric practices. This could constitute a care plan tailored for frail people with a psychiatric illness, so as to improve the outcomes of their treatment and quality of life.

Keywords: ageing well; frailty; health care; mental health; older people; resilience 


\section{Introduction}

Frailty is an age-related condition of declining functional reserves against stressors, resulting in vulnerability to negative health outcomes such as; increased risk of mortality, disability, falls, and hospitalisation/institutionalisation (Fried et al., 2001). The condition is independently associated with morbidity and disability (Clegg et al., 2013, Fried et al., 2001) and the condition becomes more prevalent with a globally ageing population ( $\mathrm{He}$ et al., 2016). The traditional definition of frailty focuses chiefly on physical aspects, and is usually considered as a stereotype of older people, who are considered physically incapable and disengaging from society (Gilleard and Higgs, 2013). The stereotype influences the way society treats older people, and the way older people see themselves (Grenier, 2007). Instead of considering merely the negative quality of frailty, an approach with multidimensional, and positive stance such as; assessing an older persons' capacity, strength, and adaptation would lead to more optimistic and practical interventions (Gobbens et al., 2010, Nicholson et al., 2017, Rodriguez-Manas et al., 2013).

Additionally evidence about the association between frailty and some psychiatric, or mental illnesses in older people, for example; dementia, depression, and delirium, has been growing rapidly in recent years (Brown et al., 2016, Sampson, 2012, Vaughan et al., 2015, Verloo et al., 2016). The prescription of some psychotropic drugs, for example antipsychotics and antidepressants, is also related to increasing the risk of frailty (Curkovic et al., 2016). However, there is currently little evidence which recognises changing perspectives of frailty in psychiatric clinical practice.

In the UK National Health Service (NHS), to access mental health services, older people with mental health problems need a referral, attached to a medical history, from their general practitioners or Acute and Emergency unit doctors. When they enter mental health services, they will usually receive a detailed assessment by a multidisciplinary team, including; a psychiatrist, occupational therapist, social worker, psychologist, and nurse. This detailed assessment will cover a wide range of issues, in order to gain an accurate picture of the individual (NHS, 2016). This could be mapped onto a Comprehensive Geriatric Assessment (Cesari and Theou, 2017) and inform the frailty status of the patient. However, there is limited evidence about how frailty is specifically assessed within the services. 
In this study we aim at exploring the current experiences, perspectives, and expectations of a sample of psychiatrists. The research questions for this study are: whether psychiatrists are familiar with frailty and frailty assessments; how frailty is relevant to and assessed in psychiatric practice; and how a frailty assessment can be successfully implemented in psychiatric practice.

\section{Methods}

\section{Data collection method: semi-structured interview}

This research was underpinned in the epistemology of constructivism and theoretical perspectives of interpretivism. The perspectives of the psychiatrists were approached with methodological perspectives of phenomenology. This was based on individual, semistructured interviews. The interviews were guided by a topic guide developed based on the research questions, literature, and discussions among the researchers (KA and AT). The topic guide covered a range of areas including; current practice in caring for older adults, familiarity with concepts and assessments of frailty, specific concerns about frailty in older adults with mental health problems, and potential challenges of introducing a formal frailty assessment in psychiatric practice.

\section{Sampling strategy and sample recruitment}

The population of the study were UK psychiatrists, who were involved in the care of older people. It was planned to conduct 8-12 interviews of approximately 30 minutes in length. A 'snow-balling' technique for the sampling strategy was adopted. It is a part of the purposeful sampling method, which ensures that the selected respondents can provide valid and relevant information. Additionally, to ensure a variety of viewpoints being included maximum variation sampling was adopted. This was accomplished by recruiting psychiatrists holding different positions, from trainees to consultants. The junior psychiatrists could compare their recent experiences both in psychiatry and general/geriatric medicine. While the senior psychiatrists could provide more practical and concise experiences related to the care of older people with frailty.

To create 'snow-balling' sampling, the researcher (KA) used both his working and social networks within the UK. The researcher's colleagues were asked to send out the research invitation letter along with information sheet to psychiatrists in the UK, who were not prior known to the researchers. At the end of each interview, the respondents themselves 
were asked by the researcher to send out the research details to their own networks. The inclusion criteria were: being a psychiatrist either a trainee or consultant; caring for older people with psychiatric problems; and working in any psychiatric settings in the UK. The interviews were conducted at a mutually convenient date, time and venue.

Ten psychiatrists participated in this research; five males and five females. The mean age of the respondents was; 36.9 years $(S D=9.1)$, ranging from $27-54$ years. In this group, there were three core trainees, five specialised trainees and two consultants. All respondents were working in NHS mental hospitals, located in London or nearby cities. All interviews were conducted by the researcher (KA) from June to July 2018, taking 19-50 minutes in length (mean $=30$ minutes, $\mathrm{SD}=9$ ).

\section{Ethical considerations}

This research complies with the ethical standards of the relevant national and institutional committees on human experimentation and with the 1964 Helsinki declaration and its later amendments or comparable ethical standards. All procedures involving human subjects were approved by the King's College Research Ethics Committee; reference number was MRS-17/18-6212. The respondents were asked for permission for the researcher to make an audio-record of the interview for the purpose of data analysis. All audio files as well as resulting transcriptions were kept confidentially, anonymised and stored in a secure place. Written informed consent was obtained from all respondents before the researcher started collecting data.

\section{Data analysis}

All interview schedules were transcribed, verbatim. The interview schedules, notes and transcriptions were repeatedly reviewed by the researchers (KA, AT), so as to gain a deeper understanding of information (Pope et al., 2000). A thematic analysis method was adopted, using a traditional hand-coding technique and NVivo software. The transcriptions were coded in order to explore initial themes and subthemes. To increase creditability, an audit trail was created along with the analysis (Mays and Pope, 2000). This was to reflect the ongoing process of identifying themes by, describing how an individual discusses the topics, comparing information across respondents and relating all these to find the connections, and thus form themes. All themes and subthemes were reviewed, and refined by both researchers 
before finalising them. The data were presented in thematic descriptive style, based on the emerging themes.

\section{Results}

\section{General themes}

From the thematic analysis, there were eight initial themes emerging from this study, regarding the research questions. These were further grouped into two main themes; 1) concept of frailty from the respondents' perspective and 2) frailty assessment in psychiatric practice in the UK. The following findings and discussion are structured around themes that were mentioned by respondents.

\section{Concepts of frailty from the respondents' perspectives}

Frailty seemed to be an ill-defined term, which had some different aspects from general medical practices. Although frailty is generally related to a negative stereotype, it could be reframed in a more positive sense via a multi-dimensional approach.

\section{- 'ill-defined term, predominantly in physical and cognitive'}

From many psychiatrists' opinions, frailty lacks a clear definition, or ways to tackle it, and is mostly used in geriatric medicine. The term needed to be studied, so as to establish a common understanding. Some psychiatrists viewed it as merely a non-specific term to describe people which might not be measured formally.

All the psychiatrists said that frailty originally involved physical and cognitive dysfunctions. The main dysfunctions were referred to; functional disability, mobility, multiple comorbidity, polypharmacy and cognitive impairment. Some respondents mentioned nutritional status. Functional disability was most frequently referred to when discussing the concept. Some respondents would describe frailty as not different from disability in terms of psychiatric clinical use.

In different cultures, expectations of older people's health, functions as well as thresholds of what is abnormal is greatly different. An equivalence of the word 'frailty' in different languages would represent the condition in different ways. Some said the term might be difficult to be used in academic communication globally.

\section{- 'multidimensional in nature'}


From most respondents' perspective, frailty would be influenced a great deal by psychological, spiritual and social factors. Depression, fear and anxiety, loneliness, loss of motivation as well as self-perception of losing identity, autonomy and meaning of life would affect older people's functions and quality of life, or vice versa.

It was felt that people living in a lower socioeconomic status would be at risk of lifelong, poor health behaviours, for example; smoking, alcohol drinking, substance abuse, physical inactivity and poor diet control. These caused multiple comorbidities in later life. Older people in different generational cohorts, might be impacted by different factors. Social situations across a lifetime gave a unique meaning to each cohort. Some older people lived independently. They were considered to be at risk of loneliness, poor health monitoring and inadequate in-home safety. Some respondents worried that some frail, older people were left socially isolated due to it being seen as a normal situation.

“...I guess if a person is...considered frail...people might think it's more acceptable that they are socially isolated..." (P08)

Some respondents commented that current care is too systematised, and is preoccupied with completing reports. Enabling and maximising functional capacity of older people, despite physical disabilities, would be more humane and holistic.

\section{- 'something different for frailty in psychiatric patients'}

In some of the respondents' opinion, organic disorders, such as; dementia and other cognitive impairments might be more relevant to frailty, than functional ones, such as; schizophrenia, depression and anxiety disorders. Psychiatric conditions were usually unstable, having multiple on and off episodes. Some respondents suggested frailty in psychiatric patients should consider levels of frailty at different time-points.

Older people with mental disorders could suffer from compulsory admission, which is a specific circumstance in mental health services. This situation leads to negative feelings, and a sense of lost autonomy. The admission of acute episodic of mental symptoms is usually longer than admission from a general ward. This could affect their functional capacity, mobility, psychological wellbeing as well as disrupt their social contacts. During long admissions, the frequent turnover of staff, having different approaches would even cause distress and confusion for older people. After discharge, they might also have some residual symptoms, causing difficulty for informal and formal carers. 
“...the patients that are frail...with psychiatric backgrounds, often, they're not able to hold down, onto any carer, or caring package...because it would be difficult to work with..." (P09)

In respondents' opinion, older people with long-term mental illness were at risk of decreased social networks, especially family and friends. They might have some conflicts with neighbours, which in turn causes them tenancy difficulties.

Respondents also referred to some research suggesting that some psychiatric medications prescribed to older people, with mental disorders, makes them even more frail at a quicker rate. It was also interesting that one respondent regularly saw older people, who had lifelong learning disabilities, eventually became older and more frail. This was a special group of frail, older people.

\section{- 'negative labelling, affecting care'}

Some respondents did not view the word 'frailty' as a pejorative term, as normally they would need a word to call certain phenomenon, communicate between colleagues and manage it. Additionally, a few respondents considered that some terms in psychiatry, such as; dementia, depression, and schizophrenia, were more stigmatised. However, most respondents were concerned about its negative quality, mirroring a stereotype of older people.

"...the public perception about what it means to be frail... of the wizened old lady bent over her cane..." (P07)

From some respondents' experience, their older patients did not like to be labelled as frail. Some even rejected treatment, help and living arrangements, such as; walking aids, which visibly mirroring the negative stereotype. They feared being transferred to residential, or nursing homes. Professionals were often too rushed to determine that some older people were frail, and offered help they did not need. This could upset them.

“...sometimes...we almost treat them too frail when they're not...just being aware that people have varying levels... allowing patients space to voice how they can be supported without feeling... a burden...we just rush so much..." (P03)

Indeed, this negative stereotype, of frail older people, could lead to a problem of 'therapeutic nihilistic'. Frail people would be considered as impossible to cure. This could influence health professionals' levels of effort on devising a care plan, and acting upon them. 
“...there is still a lot of therapeutic nihilism...people are not treated because they are old for certain conditions...[now]...it's no longer...[for]...because you are old, but you are allowed to say I'm not treating this because you're frail...” (P08)

\section{- 'preventable and reversible with older people's resilience'}

Substantial numbers of respondents considered frailty as a preventable and reversible condition, as there was an obvious disparity between, chronological age and their actual health status, indicating their biological age. Many respondents referred to; biological resilience, behavioural adaptation, social participation, and recalling of previous abilities of frail people, in order to maintain their own well-being.

“...he's ninety-nine...every day he goes to local high street...goes shopping...makes cakes and plays bridge... an amazing life...it's about finding out what people can do within the limits of their physical..." (P03)

One of the emerging issues was the involvement of older people with mental illness in devising care plans, especially those who would eventually develop frailty and lose decision making capabilities. Advanced care plans, discussed with the patient should be emphasised. Even in the later stage, the family or carers, who are the decision makers and know the best interests of the patient, should be well-informed, trained and allowed to express their voice.

\section{Frailty assessment in psychiatric practice in the UK}

Formal, frailty assessments for older people in psychiatric practices might not be in place, but there were some assessments which regularly conducted could be used for evaluating frailty status. To increase formality in frailty assessment many challenges have to be overcome, and need more evidence to support them. In the meantime, we could assess frailty with a more positive stance, which also requires a lot of changes in the current practices and attitudes of psychiatrists.

\section{- 'equivalences of formal frailty assessment'}

Regarding psychiatric settings, all respondents explained that there was no full frailty assessment in place. However, as a multidisciplinary team (MDT), each health professional might assess certain domains of frailty. The respondents explained that occupational therapists regularly assessed functional capacity and living circumstances. Social workers 
assessed social support and provided social aids. Nurses assessed physical and mental health during admission. Older patients' nutritional status was also assessed by the team, and were referred to dieticians, if needed. The psychiatrists, receiving the medical history from the GP, would assess older people on mental health status, or sometimes physical problems. These could indicate the frailty status of the patients. However, some respondents reported that there were some problems in sharing such data, for example; complicated reports for psychiatrists to review in limited time, and even sometimes no chance to review such reports.

Most of respondents would flag up frailty assessment, and interventions for their patients, with some clinical signposts or personal 'gut-feeling'. For example; they might witness patients entering the room with difficulty, or using walking aids, having history of multiple comorbidity, polypharmacy and unprecedented falls. These would signal the respondents to activate some further actions.

\section{- 'challenges of frailty assessment in psychiatric practice'}

To include a formal frailty assessment in psychiatric practice might encounter resistance from staff as there are many clinical forms to fill in along with time constraints. Some respondents called for more clear evidence of benefits. Then they might be willing to change their current practices. Although the assessment could be tailored to a shorter version, the short assessment form might be too simple, and neglect a specific context for older people.

"...the form we have is very naive... basically a little form to tick..." (P04)

Some respondents mentioned psychiatrists in general might feel uncomfortable with tackling physical illness. They sometimes left the issue to a GP, or other specialties. However, some respondents reported the referral gap between mental health and physical health services, due to separate trusts and care managements in certain settings.

“...we end-up sending our patients to A\&E along with everyone else, which seems ridiculous...they're inpatients in a hospital but...have to go to A\&E for assessment to decide whether to admit them..." (P03)

There was often some frustration in MDT meetings to assign a sponsor for social and mental health care. As criteria for eligibility of the care, from each stakeholder's perspective could at times not reach an agreement. 
"...the criteria...I have for what should be provided and the criteria...the local authority have, are completely different..." (P04)

\section{- 'diplomatic ways to approach frailty'}

As frailty is generally a stigmatised term, most of the respondents did not usually use the term directly with patients. Instead. they assessed frailty in a 'more diplomatic', or polite way.

"... a diplomatic way by asking the questions in indirect way...not using terms they would find upsetting or offensive...introducing it slowly... a bit of pieces..." (P04)

Some respondents suggested that evaluating patients' not only for disability but also ability, in order to provide some interventions, enabling them to stay physically active, participate in social activities, and enjoy the fullest life. Some respondents even suggested using an 'ability assessment form'.

Regarding the respondents' perspective, a public campaign to promote understanding of frailty was needed to de-stigmatise, and normalise the term. This could be adapted from campaigns for many mental disorders which have been done in the past. Medical and psychiatric training could adopt a more holistic approach to older people, with a balance between bio-psycho-social-spiritual concerns. Some respondents stated that there was already an increase in research and health campaigns related to frailty. This was an opportunity to study, and change care for frail people into more positive and integrated ways.

\section{Discussion}

\section{Concepts of frailty}

From this study, it seemed that some psychiatrists in the UK might not be fully familiar with the multidimensional nature of frailty. Some recognised it merely on physical and cognitive dimensions. The concept can be very different across society and professionals which causes even more confusion (Grenier, 2007, Rodriguez-Manas et al., 2013).

Consistent with most respondents' views, psychiatric disorders and psychological symptoms can undermine older people's overall health and functions due to their effects on declining physical functioning and loosening social networks (Kiecolt-Glaser et al., 2002). Psychiatric illness causes frailty both directly and indirectly, via biological and behavioural mechanisms. All of the respondents were concerned that psychiatric medications prescribed 
by psychiatrists can cause multiple metabolic diseases, immobility and cognitive impairments resulting in frailty (Curkovic et al., 2016), especially in the long-term. The respondents suggested close monitoring of this.

From the respondents' perception, the psychosocial and spiritual aspects are critical to the concepts of frailty. Frail older people may suffer spiritually from losing their sense of identity, dignity and autonomy due to their general appearance and inability to continue their normal activities (Lloyd et al., 2016). This is further worsened by negative stereotypes from society about being frail. Negative stereotypes could prevent frail people from proper and timely treatments (Nicholson et al., 2012). This was also recognised by psychiatrists in this study. Unfavourable social circumstance such as limited social support and perilous environments can put older people at risk of developing or worsening frailty (Kiecolt-Glaser et al., 2002, Tinker and Ginn, 2015).

Regarding psychiatric patients, the respondents pointed out that some long-term as well as compulsory admissions are unavoidable for certain conditions to provide proper treatment and prevent harm to themselves and others. Those admissions could cause older people feelings of distress, and a sense of losing autonomy and loosening social relationships (Fillit and Butler, 2009, Lloyd et al., 2016). In addition to the common stigmatisation of having mental health problems, which was mentioned by psychiatrists in this study, patients can feel alienated in returning to their normal life (Parcesepe and Cabassa, 2013).

Consistent with the homeostatic concepts, frailty in the perceptions of respondents can be preventable and reversible by tackling various dimensions of bio-psycho-social-spiritual (Clegg et al., 2013, Gobbens et al., 2010). We may not only treat older people's diseases, but also activate their abilities and train them for new adaptive skills. This is to maintain their overall quality of life against frailty.

\section{Frailty assessment in psychiatric practice}

There is a recommendation that frailty assessment should be included in all health care settings involved in older people (Turner et al., 2014). However, from the respondents' views, there might not be a formal frailty assessment in place in psychiatric practice as the assessment had been done at the primary healthcare services and/or other specialties. This may be due to the traditional concept of frailty focusing chiefly on physical aspects. 
However, the respondents acknowledged that a large number of assessments were regularly performed within psychiatric practice. The assessments were done by a variety of professionals and brought to the MDT meeting to devise care plans and budgets. These covered the domains of instrumental activities of daily living (iADLs), basic ADLs (bADLs), social networks, cognitive and mental functions, history of falls, gait/balance status, and nutrition. Regarding the frailty index, the results from the comprehensive assessments could be mapped onto the formal frailty assessment and the frailty index calculated without extra instruments (Searle et al., 2008). Some respondents mentioned about clinical signposts, for example medical history and patients' presentation, which could alert some further specific assessments (Chen et al., 2018, Kamhi, 2011). There was substantial evidence showing the benefits of establishing frailty assessment from routine practice and databases which would minimise the burden of implementation (Gilbert et al., 2018). However, this needs further study and validation before using in psychiatric practice.

The comprehensive assessments could subsequently inform an integrated care plan considering every aspect of patients and coordinating various services tailored to patients' needs. The concept of integrated care, to decrease treatment gaps, showed significant effectiveness in caring for frail older people with mental disorders as noted in previous studies (Goodwin and Lawton-Smith, 2010). Although integrated care is a standard in older adult services, the treatment gaps between mental health and physical health services were repeatedly reported by respondents in this study. By improving the integrated care, older people may be helped to use their reserves against frailty.

Although mental health services are now available on the basis of need regardless of age, older people suffer from discrimination in mental health services with substantial evidence of inaccessibility of certain services (RCPsych, 2009a). This was also mentioned by the respondents of this study. For example, one respondent reported that there are therapeutic nihilistic perceptions, or skeptism about possibility to cure and intervene, regarding frailty in older people among professionals.

As the respondents' responses showed, a humane, and respectful, but transparent, and informative approach to frailty, can help the assessment run smoothly (Grenier, 2007). Additionally, an exploration of frail people's ability could lift both psychological and spiritual well-being, despite their physical decline. Many respondents always checked with their older adult patients about their living conditions. Thorough evaluation of frequently 
overlooked issues such as environmental conditions and social circumstances could inform some interventions to help them live in community securely, feel confident to connect with society and foster inter-dependence (Tinker and Ginn, 2015).

Concerns about an inadequate clinical training and research on frailty in the contexts of psychiatric practice were raised by the respondents. If healthcare professionals lack understanding about certain conditions, they could feel incompetent which could prevent them from providing certain services (Kamhi, 2011). Better understanding and awareness of frailty among psychiatric professionals may not be gained, unless there is enough clinical training and evidence from research. The enriched knowledge and positive attitudes on frailty could bring to public awareness and de-stigmatisation of frailty, which eventually may enhance accessibility of older people to mental health services and frailty assessments (BritainThinks, 2015, Grenier, 2007).

\section{Strengths and limitations of this research}

To the researchers' knowledge, this was the first study on the perspectives of psychiatrists on frailty assessment. It is based on the inductive approach, to gather rich, meaningful and contextual information. The population of the study were psychiatrists, who could offer some insights related to psychosocial aspects that may enlighten medical practices and the existing concept of frailty.

There is also the possibility of having selection bias, due to using the snowballing technique in which the respondents were recruited from just certain networks and might have similar backgrounds. This research may not represent whole UK psychiatrists. However, the research was successful in recruiting a variety of respondents concerning; age, gender and positions, with no one having had a prior relationship with the researchers. These could enhance representativeness and minimise selection bias (Gray, 2018).

The researcher applied some 'trustworthiness' strategies to increase study rigour. For example, an audit trail was created and tracked throughout the analysis. Data collection from different backgrounds of psychiatrists was a data triangulation. The 'multiple voices' of every respondent, which might contain subtle and conflicting accounts, were considered (Mays and Pope, 2000). 


\section{Reflexivity}

Regarding epistemological reflexivity, this research was grounded with the multidimensional and homeostatic concepts of frailty, which viewed frailty as; a dynamic, reversible condition via multiple factors, including individual ability. This could influence the approach of the researcher, via questions, and lead the respondents in the same direction of the research.

Concerning personal reflexivity, all respondents fully engaged during the interviews. However, as the researcher himself (KA) is a psychiatrist, sharing an interest in Old Age psychiatry, the researcher, sometimes, unintentionally expressed his opinions about frailty in older people, thus, to some extent possibly affecting the respondents' responses to some questions. This was when the researcher had to consciously remind himself that he was there to consider respondents' perspectives, not influencing, or evaluating this or their knowledge. However, in some cases the interviews were more interactive, to make the interview to be more like a shared conversation (Gray, 2018).

\section{Implications and future research directions}

It is hoped that this research could inspire some psychiatrists to focus on frailty for their older patients. Further study could address some specific concerns of psychiatric patients. These included effects on frailty of long-term and compulsory admissions, episodic features of the clinical course, long-term psychiatric medication use, effects of advance care plan on frailty, limited social networks resulting from mental illness and dual stigmatisation of having mental disorders and frailty. The studies would gain much insight if done across different care settings and cultures and involving older people and their carers' voices.

A clear definition and benefits of a frailty assessment within psychiatric practices is still questioned by psychiatrists, before integrating them into their routine practice. However, psychiatrists should be empowered to explore these along with other professionals, as they have been trained in approaching bio-psycho-social-spiritual issues of people that seems to fit the multi-dimensional concepts of frailty (Brown et al., 2016, Gobbens et al., 2010, RCPsych, 2009b). Indeed, they could help refine the concept. By doing all of these we could gain a more positive, holistic and practical definition of frailty, which can lead to better understanding and awareness of older people with frailty. 
It is also hoped that the study will help other health care and social care providers and professionals to understand the views of psychiatrists about frailty. It may also help everyone who works with older people.

\section{References}

BritainThinks 2015. Frailty: language and perceptions a report prepared by BritainThinks on behalf of Age UK and the British Geriatrics Society. In: BRITAINTHINKS (ed.). Age UK, the British Geriatrics Society.

Brown, P. J., Rutherford, B. R., Yaffe, K., Tandler, J. M., Ray, J. L., Pott, E., Chung, S. \& Roose, S. P. 2016. The Depressed Frail Phenotype: The Clinical Manifestation of Increased Biological Aging. Am J Geriatr Psychiatry, 24, 1084-1094.

Cesari, M. \& Theou, O. 2017. Frailty: The Broad View. In: FILLIT, H. M., ROCKWOOD, K. \& YOUNG, J. (eds.) Brocklehurst's Textbook of Geriatric Medicince and Gerontology. 8 ed. Philadelphia, PA: Elsevier, Inc.

Chen, C. Y., Gan, P. \& How, C. H. 2018. Approach to frailty in the elderly in primary care and the community. Singapore Med J, 59, 240-245.

Clegg, A., Young, J., Iliffe, S., Rikkert, M. O. \& Rockwood, K. 2013. Frailty in elderly people. Lancet, 381, 752-62.

Curkovic, M., Dodig-Curkovic, K., Eric, A. P., Kralik, K. \& Pivac, N. 2016. Psychotropic medications in older adults: a review. Psychiatr Danub, 28, 13-24.

Fillit, H. \& Butler, R. N. 2009. The frailty identity crisis. J Am Geriatr Soc, 57, 348-52.

Fried, L. P., Tangen, C. M., Walston, J., Newman, A. B., Hirsch, C., Gottdiener, J., Seeman, T., Tracy, R., Kop, W. J., Burke, G., McBurnie, M. A. \& Cardiovascular Health Study Collaborative Research, G. 2001. Frailty in older adults: evidence for a phenotype. J Gerontol A Biol Sci Med Sci, 56, M146-56.

Gilbert, T., Neuburger, J., Kraindler, J., Keeble, E., Smith, P., Ariti, C., Arora, S., Street, A., Parker, S., Roberts, H. C., Bardsley, M. \& Conroy, S. 2018. Development and validation of a Hospital Frailty Risk Score focusing on older people in acute care settings using electronic hospital records: an observational study. Lancet, 391, 1775-1782.

Gilleard, C. \& Higgs, P. 2013. The fourth age and the concept of a 'social imaginary': A theoretical excursus. J Aging Stud, 27, 368-76.

Gobbens, R. J., Luijkx, K. G., Wijnen-Sponselee, M. T. \& Schols, J. M. 2010. Towards an integral conceptual model of frailty. J Nutr Health Aging, 14, 175-81.

Goodwin, N. \& Lawton-Smith, S. 2010. Integrating care for people with mental illness: the Care Programme Approach in England and its implications for long-term conditions management. Int J Integr Care, 10, e040.

Gray, D. E. 2018. Doing Research in the Real World, London, UK, SAGE Publications Ltd.

Grenier, A. 2007. Constructions of frailty in the English language, care practice and the lived experience. Ageing \& Society, 27, 425-445.

He, W., Goodkind, D. \& Kowal, P. 2016. An Aging World: 2015 International Population Reports. Washington, DC.: U.S. Census Bureau.

Kamhi, A. G. 2011. Balancing certainty and uncertainty in clinical practice. Lang Speech Hear Serv Sch, 42, 59-64.

Kiecolt-Glaser, J. K., McGuire, L., Robles, T. F. \& Glaser, R. 2002. Emotions, morbidity, and mortality: new perspectives from psychoneuroimmunology. Annu Rev Psychol, 53, 83-107.

Lloyd, A., Kendall, M., Starr, J. M. \& Murray, S. A. 2016. Physical, social, psychological and existential trajectories of loss and adaptation towards the end of life for older people living with frailty: a serial interview study. BMC Geriatr, 16, 176.

Mays, N. \& Pope, C. 2000. Qualitative research in health care. Assessing quality in qualitative research. $B M J, 320,50-2$. 
NHS. 2016. How to access mental health services [Online]. National Health Service. Available: https://www.nhs.uk/NHSEngland/AboutNHSservices/mental-health-servicesexplained/Pages/accessing\%20services.aspx [Accessed 2 August 2018].

Nicholson, C., Gordon, A. L. \& Tinker, A. 2017. Changing the way "we" view and talk about frailty. Age Ageing, 46, 349-351.

Nicholson, C., Meyer, J., Flatley, M., Holman, C. \& Lowton, K. 2012. Living on the margin: understanding the experience of living and dying with frailty in old age. Soc Sci Med, 75, 1426-32.

Parcesepe, A. M. \& Cabassa, L. J. 2013. Public stigma of mental illness in the United States: a systematic literature review. Adm Policy Ment Health, 40, 384-99.

Pope, C., Ziebland, S. \& Mays, N. 2000. Qualitative research in health care. Analysing qualitative data. $B M J, 320,114-6$.

RCPsych 2009a. Age discrimination in mental health services making equality a reality. In: UNIT, T. C. S. O. A. P. F. A. P. (ed.) Royal College of Psychiatrists' position statement. The Royal College of Psychiatrists.

RCPsych 2009b. Good clinical care. Good psychiatric practice. 3 ed. Dorchester: The Royal College of Psychiatrists.

Rodriguez-Manas, L., Feart, C., Mann, G., Vina, J., Chatterji, S., Chodzko-Zajko, W., GonzalezColaco Harmand, M., Bergman, H., Carcaillon, L., Nicholson, C., Scuteri, A., Sinclair, A., Pelaez, M., Van der Cammen, T., Beland, F., Bickenbach, J., Delamarche, P., Ferrucci, L., Fried, L. P., Gutierrez-Robledo, L. M., Rockwood, K., Rodriguez Artalejo, F., Serviddio, G., Vega, E. \& group, F.-C. 2013. Searching for an operational definition of frailty: a Delphi method based consensus statement: the frailty operative definition-consensus conference project. J Gerontol A Biol Sci Med Sci, 68, 62-7.

Sampson, E. L. 2012. Frailty and dementia: common but complex comorbidities. Aging Ment Health, $16,269-72$.

Searle, S. D., Mitnitski, A., Gahbauer, E. A., Gill, T. M. \& Rockwood, K. 2008. A standard procedure for creating a frailty index. BMC Geriatr, 8, 24.

Tinker, A. \& Ginn, J. 2015. An age friendly city- how far has London come? London: King's College London.

Turner, G., Clegg, A., British Geriatrics, S., Age, U. K. \& Royal College of General, P. 2014. Best practice guidelines for the management of frailty: a British Geriatrics Society, Age UK and Royal College of General Practitioners report. Age Ageing, 43, 744-7.

Vaughan, L., Corbin, A. L. \& Goveas, J. S. 2015. Depression and frailty in later life: a systematic review. Clin Interv Aging, 10, 1947-58.

Verloo, H., Goulet, C., Morin, D. \& von Gunten, A. 2016. Association between frailty and delirium in older adult patients discharged from hospital. Clin Interv Aging, 11, 55-63.

Acknowledgements: The researchers would like to thank all respondents in the study. We would also like to thank all colleagues who helped in recruiting respondents. However, their names could not be disclosed due to confidentiality concerns of the respondents.

Funding: There was no funding to this research.

Conflict of interest: None 